\title{
Archives de la SNCF et patrimoine ferroviaire
}

The SNCF Archives and Railway Her itage

Henri Zuber

\section{OpenEdition}

\section{Journals}

Édition électronique

URL : https://journals.openedition.org/rhcf/711

DOI : 10.4000/rhcf.711

Éditeur

Rails \& histoire

Édition imprimée

Date de publication : 1 novembre 2009

Pagination : 25-31

ISSN : 0996-9403

\section{Référence électronique}

Henri Zuber, "Archives de la SNCF et patrimoine ferroviaire », Revue d'histoire des chemins de fer [En ligne], 40 | 2009, mis en ligne le 01 novembre 2011, consulté le 22 avril 2022. URL : http:// journals.openedition.org/rhcf/711 ; DOI : https://doi.org/10.4000/rhcf.711 
Henri ZUBER

\title{
Archives de la SNCF et patrimoine ferroviaire
}

\begin{abstract}
question de leur double statut. En effet, les archives sont à la fois le support de l'information opérationnelle courante et rétrospective et, à ce titre, apportent sur notre sujet une documentation de première main, et, par ailleurs, elles constituent en tant que telles un objet de patrimoine.

La particularité d'un service intégré comme celui que je dirige à la SNCF, le service des archives et de la documentation, est de se fixer comme objectif d'être présent tout au long du cycle de vie des dossiers et des documents. La mission de ce service est donc d'abord de mettre l'information réutilisable à la disposition des activités de la SNCF avant de la mettre ensuite, selon le modèle classique des services d'archives publiques, à la disposition du public et des chercheurs.
\end{abstract}

Le Service des Archives et de la documentation (SARDO) de la SNCF est né en 2006 du rassemblement des centres créés dans la décennie précédente par la SNCF. Il s'est renforcé il y a peu par la création d'un pôle d'expertise au service de toutes les directions. Il anime un réseau de correspondants archives 
et développe avec celui-ci l'ensemble des outils réglementaires et nécessaires à la meilleure gestion d'un fonds d'archives relativement exceptionnel.

Ce service, qui comprend, outre les centres d'archives, un centre de documentation et d'information et un « laboratoire de micrographie » fait partie de la Direction juridique groupe.

\section{La constitution des fonds}

Le Centre des archives historiques de la SNCF situé au Mans, créé en 1995, a ouvert ses portes au public en 1996. La vocation du centre est, depuis l'origine, de rassembler l'ensemble des archives historiques de l'entreprise, à l'exception des dossiers de personnel regroupés à Béziers.

La création du centre du Mans s'inscrit dans le cadre d'une politique d'archivage impulsée dans les années 1989-1990, à l'époque où Jacques Fournier était président de la SNCF (1988-1994). En effet, c'est en janvier 1989 qu'une division des Archives est créée au sein du Service général.

À sa tête, Martine Constans, conservateur des archives, parcourt l'entreprise. Elle entreprend un important travail de recensement et d'inventaire d'archives. Parallèlement, elle regroupe des archives des directions centrales dans la halle de stockage de Souppes-sur-Loing.

En 1993, le Centre d'archives intermédiaires de Villeneuve-Prairie est le premier centre d'archives à voir le jour. Villeneuve reçoit alors des directions centrales beaucoup d'archives en vrac, non inventoriées et parfois anciennes.

Deux ans plus tard, l'ouverture du Centre du Mans autorise le transfert d'archives en provenance tant de la halle de Souppes-sur-Loing que du Centre d'archives intermédiaires de Villeneuve. Ces transferts massifs submergent alors littéralement le bâtiment de réception, ainsi que la petite équipe du Mans constituée d'environ cinq à six personnes. Quelques versements en provenance des directions régionales de Paris-Nord et Paris-Est (la SNCF compte 23 "Régions ", divisions géographiques et administratives) viennent compléter la liste déjà longue des transferts.

Le Centre du Mans accueille également, à ses débuts, les ouvrages et revues rassemblés par le Centre national de documentation de la SNCF, ainsi que ceux de La Vie du rail. Les fonds documentaires occupent ainsi les premiers mètres de rayonnages, pour atteindre actuellement le chiffre de 3 kilomètres linéaires de rayonnages!

Progressivement, les archives sont mises en rayonnages et inventoriées sur informatique, mais sommairement et sans classement, ni tri. 
Le Centre du Mans reste ensuite majoritairement "alimenté ", via Villeneuve, par les archives des directions centrales.

À partir de l'année 2000, la politique de l'entreprise, cherchant à favoriser l'accès au public d'archives de la période 1939-1945, déclenche plusieurs transferts partiels d'archives en provenance des directions régionales, telles Metz-Nancy, Reims, Lyon, etc. La masse d'archives historiques encore répartie sur l'ensemble du territoire demeure néanmoins considérable.

En ce qui concerne Béziers, le "Centre d'archives multirégional " accueille ses premières archives en 1998 et ses premiers chercheurs en janvier 2000. Béziers a la particularité d'être à la fois service d'archives historiques pour les dossiers individuels des agents de l'entreprise et service d'archives intermédiaires pour les Régions de Montpellier, de Toulouse ainsi que pour la Caisse de prévoyance et de retraite située à Marseille (CPR).

La CPR a fourni d'ailleurs à Béziers ses plus gros volumes d'archives historiques (le dossier individuel de retraite devient historique trente ans après l'extinction de la pension). Plus récemment, Béziers a accueilli les dossiers individuels d'agents, devenus historiques, conservés jusqu'alors par l'antenne CPR de Strasbourg.

Dans l'ensemble des centres, la collecte des fonds se poursuit. Au Mans, le rythme d'accroissement est en 2008 de 3 kilomètres linéaires supplémentaires par an. À Villeneuve, la décision sera prise en 2009 de doubler la capacité d'accueil des archives intermédiaires, pour atteindre un métrage total de $50 \mathrm{~km}$ linéaires.

Enfin, Béziers mettra en œuvre à compter de 2009 la dématérialisation des dossiers d'agents sortis des cadres de la SNCF, reprise d'une politique dont le SARDO sera bientôt le seul acteur.

\section{Présentation des fonds des centres d'archives historiques de la SNCF}

Il ne peut s'agir ici que de donner un aperçu sur les ressources actuellement connues et d'exposer les lacunes les plus flagrantes. Nous vous rappelons qu'une description, tant des fonds d'archives historiques du Centre du Mans que de ceux de Béziers, est consultable sur le site internet de l'AHICF (www.ahicf.com). 
Le Centre du Mans conserve actuellement les archives "classiques" d'une entreprise : archives du conseil d'administration de la SNCF, de la direction du personnel, des finances, qu'il faut savoir utiliser pour trouver des sources en rapport avec notre sujet.

Le Centre du Mans s'efforce depuis sa création de rassembler l'ensemble des textes réglementaires, de l'époque des anciennes compagnies comme de celle de la SNCF. Ces documents traitent de tous les aspects de la maison, tant de l'organisation des services, du statut et de la gestion du personnel, des procédures comptables, que de tout ce qui touche à la sécurité. Ils seront consultés à chaque instant, source capitale et unique sur le fonctionnement de l'entreprise au quotidien jusque dans ses plus petits détails, même éphémères.

Concernant les archives techniques et scientifiques, sources de l'histoire des sciences, intéressons nous en premier lieu aux archives fournies par les trois grandes directions fonctionnelles, de l'équipement, du matériel, et du transport, à l'échelle nationale. En réalité, la connaissance de ces ensembles archivistiques est encore aujourd'hui balbutiante en partie parce que la sollicitation de la part des chercheurs reste limitée. Pour le moment seuls les plans du matériel roulant et remorqué font l'objet de demandes régulières de consultation de la part des maquettistes et modélistes. Par ailleurs, beaucoup d'archives relevant de ces fonctions se trouvent encore stockées en palettes ou n'ont pas encore été versées, parfois même alors que leur identification date des années 1990. Pour ne prendre que l'exemple de l'Équipement, qui peut intéresser les services de l'Inventaire, alors que Villeneuve accueille environ $9 \mathrm{~km}$ de dossiers de l'Ingénierie et de l'Infrastructure, il faut noter par exemple que de nombreux versements de fonds historiques restent à effectuer, dans la mesure où Le Mans n'affiche que moins d'un $\mathrm{km}$. Mais sur ce plan, de très beaux versements sont entrés ces dernières années. Je pense au fonds de la Société hydroélectrique du Midi, longtemps service, puis filiale de la SNCF. Les versements de l'Équipement des régions de Chambéry et de Strasbourg, en cours depuis le début de 2008, enrichissent nos ressources dans ce domaine.

Au niveau régional, le même découpage fonctionnel existait aussi, tant au temps des anciennes compagnies qu'ultérieurement. Les ressources du centre sont plutôt inégales : la fonction de l'Équipement est bien représentée pour le Nord, l'Est et dans une moindre mesure le Sud-Ouest (Compagnies de chemins de fer, puis Régions SNCF), mais presque complètement absente pour l'Ouest et le Sud-Est ; les fonctions du Matériel et de la Traction, ainsi que de l'Exploitation, sont quant à elles faiblement représentées, tous secteurs géographiques confondus. 
Au niveau des archives que l'on peut qualifier de stratégiques, le fonds le plus intéressant est celui de la direction des études générales, de la planification et de la recherche qui offre des dossiers relatifs à l'organisation administrative et aux réformes des structure de la SNCF, ainsi que des études prospectives. Sur le plan des archives commerciales, les ressources du centre sont relativement limitées actuellement s'il l'on excepte le fonds très particulier des affiches ferroviaires.

Pour finir ce rapide panorama par la fonction juridique, signalons : les dossiers d'accidents ferroviaires concernant les voyageurs et les dossiers d'affaires contentieuses générales. Dans les deux cas, les dossiers remontent à l'époque de la structure juridique commune mise en place par les compagnies et se poursuivent au temps de la SNCF. Un récent état des lieux dressé par une stagiaire ouvre des perspectives larges d'exploitation de ce fonds.

\section{L'accès aux archives}

Le Centre des archives historiques du Mans applique bien évidemment la législation en vigueur sur les archives publiques, avec les possibilités de dérogation prévues par la loi, selon les dispositions du Code du Patrimoine, livre II, article L 211 à 214, récemment modifiées par la loi du 17 juillet 2008 sur les archives. Cette qualité d'archives publiques des archives de la SNCF les met à la disposition de tous, une fois leur libre communication validée.

Laccès est bien évidemment tributaire du degré de traitement des archives, ainsi que de la diffusion au public d'instruments de recherche.

Au Mans, un effort particulier a été accompli, depuis l'année 2000, pour les archives de la période de la guerre 1939-1945 et cela en vue d'en favoriser l'accès au public. L'effort se place effectivement tant au niveau du traitement des archives qu'au niveau de la diffusion au public d'instruments de recherche. La liste des instruments de recherche aujourd'hui présents en salle de lecture du Centre en témoigne. Cette liste est consultable depuis septembre 2002 sur le site internet de l'AHICF. Elle est régulièrement mise à jour.

Il reste toutefois encore beaucoup de travail à accomplir, nous en sommes conscients. En effet, la majorité des archives présentes en rayonnages n'ont été inventoriées que sommairement sur base de données informatique et cette base reste à usage interne. Par ailleurs, le fait que les archives n'ont pas été classées rend leur exploitation souvent délicate. En dépit de ces difficultés, le maximum est accompli par le personnel du Centre pour satisfaire l'ensemble 
des demandes de recherche et il est rare qu'aucune information ne puisse être dégagée du fonds d'archives ou du fonds de documentation.

\section{Des archives pour quoi ?}

Quelle que soit la politique de communication menée par le service, la réalité et la régularité des versements dépendent beaucoup de la mobilisation des interlocuteurs dans les services de la SNCF. Même si l'existence de centres d'archives est un atout, par rapport à des formules d'externalisation par ailleurs fortement encadrées dans le cadre d'archives publiques, les difficultés subsistent et ne sont souvent réglées qu'à l'occasion d'un déménagement. À ce titre, on peut dire que les déménagements d'activités sont à la fois la chance et la malédiction des archivistes (et des chercheurs).

La définition des périmètres d'activité peut également constituer un écueil. En effet, les réorganisations internes, mais aussi les transferts d'activités à l'extérieur d'une entreprise, sans parler des privatisations ou nationalisations sont de véritables casse-tête pour les archivistes, qui sont souvent dans l'impossibilité d'assurer une continuité des fonds, y compris quand ces évolutions de périmètre se déroulent dans un climat consensuel et serein sur le plan social.

À titre d'exemple, depuis la séparation entre l'exploitant SNCF et RFF, propriétaire de l'Infrastructure, c'est la SNCF qui continue à gérer les dossiers d'archives pour le compte de RFF, et ce n'est que rarement le service des archives qui intervient directement dans la fourniture d'informations à RFF.

L'existence d'une formule d'archivage interne avec un réseau de trois centres d'archives et des équipes internes est donc la formule retenue par la SNCF, qui n'exclut plus, en cas de surplus temporaires, ou d'archives à faible valeur éliminables à terme, à faire appel à l'externalisation.

La conséquence en est de prévoir l'accueil, dans au moins deux des trois centres, de chercheurs au sens large. Ce qui demeure insuffisant à l'heure actuelle par rapport à la richesse de l'offre, c'est un recours à cette ressource à la hauteur des moyens mis en ouvre par la SNCF. Et pourtant, par les liens privilégiés qu'entretient le SARDO avec l'Université et avec l'AHICF, les sources disponibles sont bien connues et bien diffusées, tant en France qu'à l'étranger. Il existe donc ici un décalage que je me contenterais de constater, sans pouvoir apporter plus d'éléments d'analyse. 
En revanche, ce qui est sûr, c'est que le SARDO se doit d'affirmer, au sein d'une entreprise publique, que ses racines sont au nombre de ses valeurs de service public. C'est pourquoi il me semble peu envisageable que la direction de la Communication ne fasse pas plus régulièrement, et dans une perspective de modernité assumée sur le long terne (des années 1850 à nos jours), appel aux ressources de notre service. 
